\title{
Estudio para la elaboración de un material compuesto por fibra de mimbre (salix viminalis) y acetato de polivinilo con propiedades auto- estructurantes
}

Study for the manufacturing of a material composed by wicker fiber (salix viminalis) and polyvinyl acetate with self structuring properties

\author{
María Luz Fernández Gallardo \\ Universidad de Chile, Chile \\ marialuz.fernandez.gallardo@gmail.com
}

\begin{abstract}
Experimental investigation focused on the creation of a procedural method for the manufacturing of a material made by wicker fiber and reinforced by the fixation adhesive Polyvinyl Acetate (PVA), with the objective of increasing the natural plasticity of wicker and generate self-supporting parts which lack of an internal or external structure that may give them their final morphology. Furthermore, it aims to generate new knowledge that may improve the productive techniques and contribute to the sustainable micro-industrialization of the material.
\end{abstract}

Keywords: Mimbre; Salix Viminalis; Pva; Cad-Cam; Micro industrialización

\section{Introducción}

En Chile, el mimbre es un producto forestal no maderero que tiene una importante participación en el folklore del país, se emplea desde antaño en la fabricación de muebles y de utensilios (domésticos y decorativos). Su producción se concentra mayormente en la ciudad de Chimbarongo (localidad ubicada en la VI región) y se caracteriza por ser una actividad artesanal de bajo costo, con productos de baja duración y de naturaleza rústica.

A pesar de las intervenciones de diferentes instituciones para mejorar el conocimiento sobre el cultivo y la producción del sauce-mimbre (Salix viminalis) (apertura de mercados, capacitaciones y aplicación de tecnologías) en la actualidad, no se ha logrado revitalizar la actividad y los artesanos dedicados al mimbre no sobrepasan las 300 familias, en contraste con las 1200 que existían en la década de los 90 (Ábalos, 2005). Las causas de esta disminución son variadas y entre ellas destacan: la migración de los jóvenes y con ello el cese del aprendizaje de la actividad por transmisión entre generaciones, la escasa innovación tecnológica en los procesos, la casi nula renovación de sus morfologías y la fuerte incorporación de los polímeros en nuestros mercados (de los cuales son consumidos unos $50 \mathrm{Kg}$. per cápita a nivel nacional) (ASIPLA, 2014). Sin embargo, la última causa mencionada podría cambiar pues según la ASIPLA (Asociación Gremial de Industriales del Plástico en Chile) el consumo de plásticos ha disminuido constantemente durante los 10 últimos años, poniendo en evidencia que un importante sector de la población presenta marcadas preferencias por los productos compuestos por materiales nobles, no contaminantes y biodegradables. Es así como los consumidores buscan en el mercado productos hechos con materiales de menor toxicidad y que asimilen las cualidades morfológicas y aplicaciones de polímeros u otros de similares materiales. (McCann, 2012)

Esta nueva tendencia ha dado lugar a la toma de conciencia y compromiso por parte de algunos diseñadores y empresas sobre el concepto del diseño "forma-función", el cual se ha visto debilitado por la excesiva relación de aspectos funcionales, económicos, tecnológicos y valor de uso. Hoy, la dimensión simbólico-psicológicas cobra mayor relevancia, destacándose el predominio de la dimensión comunicativa de los objetos y los aspectos psicológicos de la relación usuarioproducto. Estos elementos constituyen un factor determinante del diseño en las últimas décadas, debido a que el objeto industrial es un soporte de comunicación que influye en la generación y definición de valores culturales. (Bañó, 2010)

La industria del mimbre reúne perfectamente las características para la aplicación de una dimensión más integradora del diseño dado su importancia cultural y debido a que se ha visto privada de la exploración de nuevas maneras de trabajo y de nuevas morfologías en sus productos, lo cual se refleja en la desactualización de los procesos de fabricación pues aún predomina el uso de estructuras internas o externas (removibles o no) como medio para dar forma al mimbre, proceso que limita la forma externa de los productos, restringiéndolas a formas cerradas y por revolución. Es por esta razón que la idea de incorporar nuevas técnicas productivas consistentes en combinaciones de materiales y 
tecnologías que resuelvan esta problemática, sin duda, daría un nuevo realce a la actividad.

El presente estudio se enfoca en identificar los fundamentos prácticos, recopilando información respecto de los parámetros y variables que sean necesarios para el moldeado y fijado de entramados de huira de mimbre para la producción de piezas de diferentes morfologías. Asimismo, se centra en explorar las capacidades morfológicas del tejido a través de la experimentación con moldes, contra-moldes y un adhesivo de fijación con el propósito de liberar al mimbre de su soporte estructural y de aprovechar y destacar su plasticidad natural al ampliar su tipología morfológica.

La investigación busca potenciar y reutilizar materiales naturales con significado socio-cultural para el país, significado que, desde una perspectiva del diseño posmoderno, considera al mimbre como una materia prima nacional y fomenta la búsqueda de nuevos procesos productivos que logren brindar aplicaciones o técnicas que lo mantengan vigente, contribuyendo así al desarrollo tanto en el área social, económica y medioambiental de la comunidad participe y en consecuencia derribando la idea que la industrialización de un producto artesanal lo lleva a una extinción segura. El diseño y la visión acerca de la identidad de los productos hacen que la búsqueda de nuevos procesos para la fabricación de un material realce tanto a éste como al oficio que lo crea.

\section{Materiales y Métodos}

Se realizó una investigación experimental que consta de dos partes. En la primera se seleccionan los materiales y se definen los parámetros y variables necesarios para la realización de las pruebas de fijación de la huira entramada. En la segunda parte se recopila información a través de pruebas de curvado del material en diferentes etapas morfológicas para su posterior evaluación.

\section{Selección de técnica y materiales}

La actividad del mimbre no ha avanzado a la par con las tecnologías ni con los procesos productivos usados en la actualidad. Al visualizar las diferentes técnicas productivas presentes en el mercado actual, para la construcción de piezas curvas en distintos materiales, se logra identificar aquellas que son posibles de introducir al mercado artesanal del mimbre. Una de ellas es la técnica del machihembrado, cuya ejecución y producción hoy en día se ve facilitada por la incorporación de tecnología CAD-CAM, tecnología que permite mejorar la eficiencia en la generación de moldes y el tiempo de construcción.

Para fines de esta investigación se utilizan moldes tradicionales constituidos por tableros de fibra de densidad media (MDF) prensados entre sí y modelados en una Router CNC (moldes sin ventilación). Así mismo, se propone una variante al molde tradicional el cual está compuesto por piezas de MDF de $3 \mathrm{~mm}$ cortadas en láser que deben ser ensambladas y encoladas en sus ranuras (moldes con ventilación)

Por su parte, la huira de mimbre presenta diferentes formatos disponibles en el mercado, entre ellas: la huira de mimbre cocido y de mimbre crudo. La selección del tipo de huirá se realiza privilegiando la componente estética del material por sobre la mecánica, pues la primera, presenta una mayor aceptación en el mercado nacional.

ETAPA N¹: Molde Solido

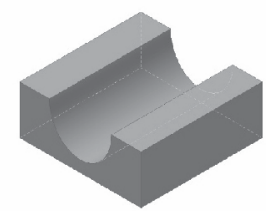

Figura 1: Tipos de moldes por etapas. Elaboración propia.

Por último, se selecciona el adhesivo PVA (Acetato de polivinilo) por sobre otros compuestos similares, debido a su bajo nivel de toxicidad medioambiental; su resistencia y mediana clasificación de biodegradabilidad, características que cumplen con el propósito de la investigación. Entre sus propiedades más destacadas se menciona su solubilidad en agua, su resistencia a ésta una vez seco y que responde a un fácil manejo y aplicación debido a que no necesita condiciones especiales de uso.

\section{Definición de las morfologías a evaluar}

El estudio se centra en responder la hipótesis que propone dar forma a una trama de mimbre sin necesidad de una estructura de sostén. Para esto, se hace necesaria la definición de morfologías que se generan a través de ángulos de curvatura que, como condición principal, presenten una deformación progresiva del segmento que la representa. La longitud de dicho segmento será constante, por tanto, no variará a medida que el ángulo de curvatura cambie, situación que se representa en la figura 2 y que se modela (de manera aproximada) por medio del sistema de ecuaciones presentado en la figura 3. La solución de este sistema de ecuaciones permite identificar las medidas " $x$ " (apertura) e " $y$ " (altura) que determinan la curva y que dependen de un determinado ángulo. A las curvas resultantes se les denominan curvas principales y se entienden como aquellas curvas que inician la construcción de todas las formas que serán parte de la experimentación. La curva tendrá una longitud mínima de 200 $\mathrm{mm}$ y determinará el tamaño mínimo para cada prueba.

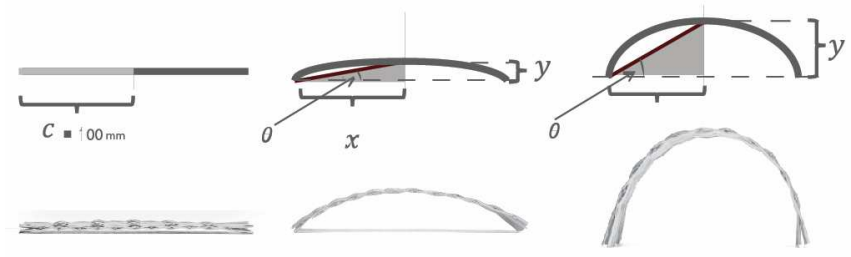

Figura 2: Segmento que representa la trama de huira e identificación de variables. Elaboración propia. 
Las curvas seleccionadas son aquellas generadas por los ángulos de $20^{\circ}, 50^{\circ}$ y $70^{\circ}$ debido a que su altura y apertura son notoriamente distintas entre sí.

Finalmente, estas curvas se les aplican movimientos de rotación, traslación y revolución para generar las morfologías finales que serán evaluadas posteriormente. La figura 4 muestra los distintos tipos de geometrías creadas por cada uno de los movimientos.

Sistema de Ecuaciones:

$$
\operatorname{tg}(\theta)=\frac{y}{x} \wedge x^{2}+y^{2}=c^{2}
$$

luego:

$$
\begin{aligned}
& \Rightarrow \quad t^{2}(\theta)=\frac{y^{2}}{x^{2}} \\
& \Rightarrow \quad t^{2}(\theta)=\frac{c^{2}-x^{2}}{x^{2}} \\
& \Rightarrow \quad t^{2}(\theta)-\frac{c^{2}-x^{2}}{x^{2}}=0 \\
& \Rightarrow \quad t^{2}(\theta) x^{2}-\left(c^{2}-x^{2}\right)=0 \\
& \Rightarrow \quad\left(t g^{2}(\theta)+1\right) x^{2}=c^{2} \\
& \Rightarrow \quad \sec ^{2}(\theta) x^{2}=c^{2} \\
& \Rightarrow \quad \sec ^{2}(\theta) x^{2}-c^{2}=0 \\
& \Rightarrow \quad\left\{\sec ^{2}(\theta) x-c\right\} \quad\{\sec (\theta) x+c\}=0 \\
& \Rightarrow \quad \sec ^{2}(\theta) x-c=0 \quad \vee \quad \sec (\theta) x+c=0 \\
& \Rightarrow \quad x=\frac{c}{\sec (\theta)} \quad \vee \quad x=-\frac{c}{\sec (\theta)} \\
& \Rightarrow \quad x=\frac{c}{\sec (\theta)}
\end{aligned}
$$

por tanto:

$$
y=\sqrt{c^{2}-\frac{c^{2}}{\sec ^{2}(\theta)}}
$$

Figura 3: Sistema de ecuaciones para la generación de curvas principales. Elaboración propia.

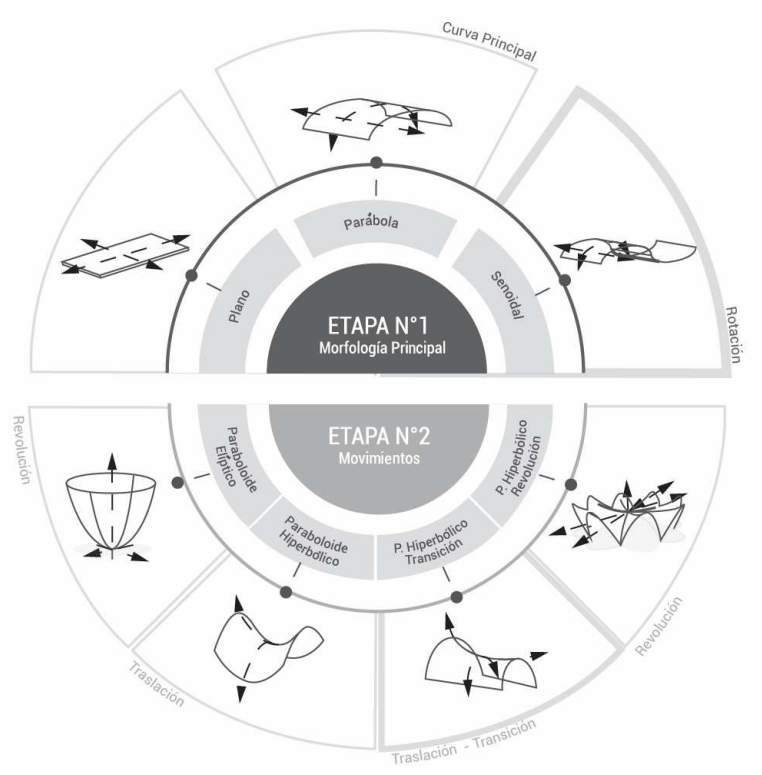

Figura 4: Morfologías a evaluar por etapas y los movimientos que las componen. Elaboración propia.

\section{Definición de variables y parámetros}

Tal como se visualiza en la figura 5, la experimentación involucra dos etapas. La primera consiste en el estudio de las morfologías con curvatura simple donde las variables a medir son: ángulo, orientación de la fibra, tipo de tejido y ancho tipo de huira. Por otro lado, la segunda etapa responde al estudio de figuras en doble curvatura, midiéndose las mismas variables que en la etapa anterior, pero conservando sólo un espesor de huira para la variable de ancho de huira. Cabe destacar que las variables que involucran el tipo de tejido y el ancho de la huira son seleccionadas a partir de los usos más comunes que se les dan en el mundo artesanal.

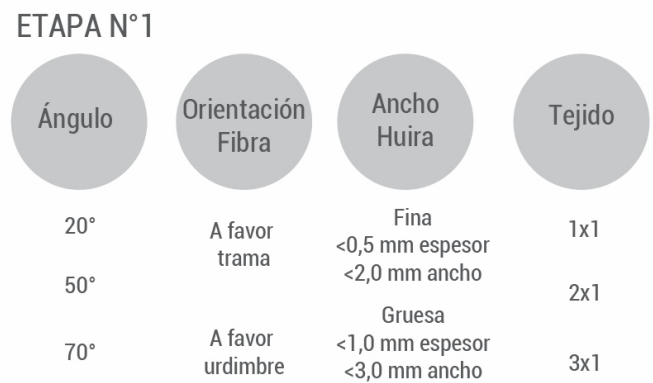

ETAPA N ${ }^{\circ} 2$

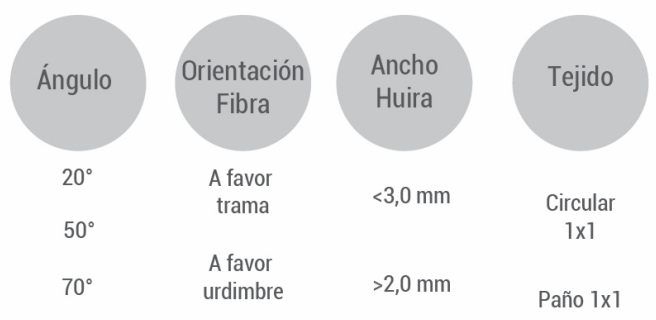

Figura 5: Variables para cada una de las etapas de experimentación. Elaboración propia. 
Por otro lado, los parámetros que se definen son el tiempo de remojo y la proporción de PVA/Agua, solución que servirá de fijador, a las que se someterán las pruebas. Para ello se realizan estudios de absorción y dilución de PVA sobre tramas de huiras de mimbre:

Absorción: Busca definir el tiempo de saturación de las fibras según su espesor para así definir un tiempo de remojo mínimo durante el cual se sumergirán las probetas.

Durante la evaluación de absorción, se pesa en seco una determinada cantidad de huiras gruesas y finas de igual longitud y se sumergen en un recipiente con agua, midiendo su peso cada 5 minutos durante de 60 minutos.

Dilución de PVA: Pretende determinar la cantidad óptima de dilución adhesivo PVA/Agua necesaria para lograr una mezcla homogénea del material de manera que el adhesivo sea absorbido por la fibra sin acumulaciones externas o notorias y a su vez sea capaz de fijar la fibra el entramado. El procedimiento consta de 18 probetas, de las cuales la mitad está fabricada con huira gruesa y la otra con huira fina. Se elaboran 9 mezclas de PVA con un peso máximo de $100 \mathrm{gr}$ cada una, variando las concentraciones de PVA cada $10 \mathrm{gr}$ (10/90 PVA/Agua hasta 90/10 PVA/Agua). Se sumergen una probeta de huira fina y una gruesa por cada una de las 9 soluciones durante un determinado tiempo. Finalmente, una vez secas, se evalúan según homogeneidad y fijación.

\section{Método}

Para la experimentación del estudio de morfologías, se dispuso de tejidos a base de huira de mimbre en forma de paños (de dimensiones $250 \mathrm{~mm} \times 250 \mathrm{~mm}$ considerando excedentes) que se sumergen durante un tiempo determinado en una solución de PVA/agua, la cual dependerá del espesor de huira con el que se esté trabajando (gruesa o fina). Luego los paños impregnados son ubicados en los moldes creados para cada una de las morfologías que se evaluarán. Por último, los paños son prensados y secados a temperatura ambiente por un total de 12 horas, para luego ser desmoldados y evaluados

\section{Análisis y resultados}

Del estudio de absorción se obtiene que ambos espesores de huira saturan su capacidad al minuto 25 (de ahí en adelante su peso se mantiene constante). Esto determina el tiempo que deben estar las pruebas sumergidas en la solución PVA/Agua, logrando así acortar los tiempos de fabricación. Por otro lado, las pruebas de dilución de PVA/Agua arrojaron que la mezcla que cumple con mayor satisfacción con los criterios de homogeneidad y fijación son las de: 50\%PVA / 50\%Agua en el caso de la huira fina y $40 \%$ PVA / $60 \%$ Agua en el caso de la huira gruesa.

Respecto de la experimentación morfológica, en la primera etapa se generan un total de 27 probetas curvadas en formas simples, mientras que en la segunda etapa se obtienen un total de 18 probetas de doble curvatura. La figura 6 detalla la cantidad de probetas producidas en cada una de las etapas y las variables evaluadas.
Todas las probetas son sometidas a evaluación según los siguientes criterios:

Homogeneidad: Evalúa la uniformidad de la mezcla de PVA sobre la trama.

Textura: Capacidad del tejido de permanecer sin deformaciones (aperturas de fibra).

Morfología: Fidelidad del material a los moldes a los que se le somete.

Cada uno de los criterios se valora con una puntuación de 1 a 3 , siendo 1 el mejor evaluado y 3 el peor. De acuerdo a los criterios evaluados y del puntaje obtenido para cada una de las muestras se seleccionan aquellas con mejor resultado.

La evaluación arroja que las probetas con mayor éxito durante la etapa 1 (etapa de curvado simple) son aquellas realizadas en tejido de huira gruesa de $1 \times 1$ (con espesor de $1 \mathrm{~mm}$ y ancho de $3 \mathrm{~mm}$, ver figura 5), en ángulos de $50^{\circ}$ y con la trama a favor de la curva principal.
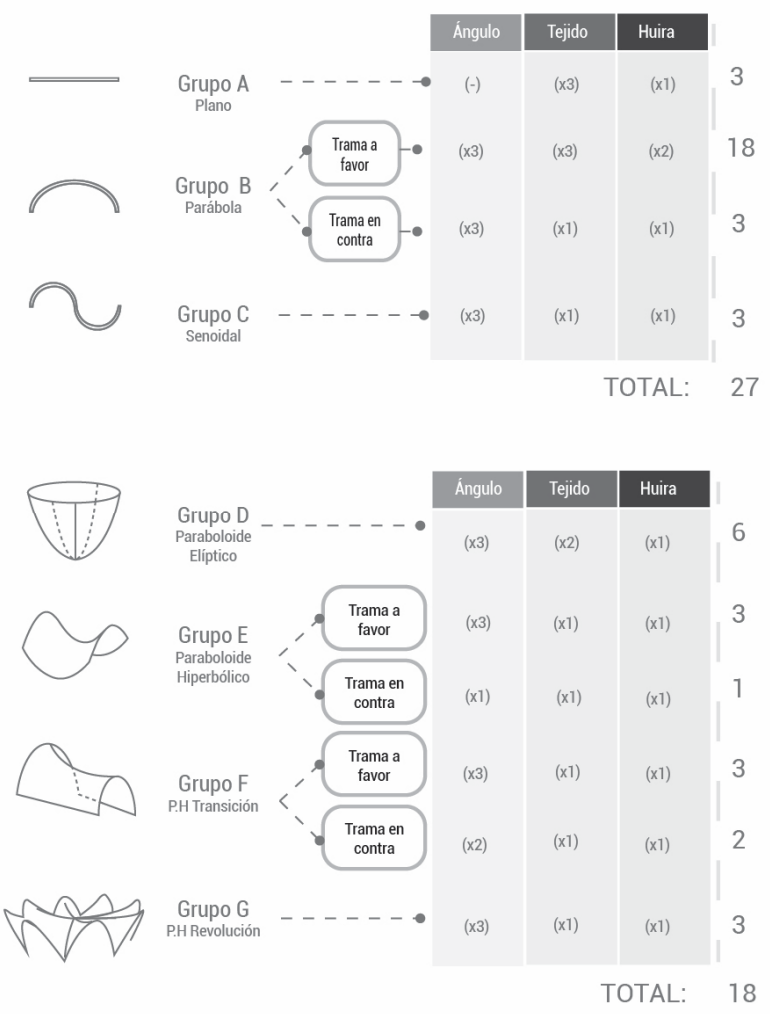

Figura 6: Cantidad de probetas según morfologías y variables. Elaboración Propia.

Por su parte, para la segunda etapa (de curvatura doble) los resultados se presentan de acuerdo a los distintos grupos morfológicos que se establecieron, los que se denominan: D (Paraboloide Elíptico), E (Paraboloide Hiperbólico o PH), F (P.H Transición) y G (P.H Revolución). 
Para el grupo D, los resultados obligan a ampliar la longitud del segmento de la curva inicial de $200 \mathrm{~mm}$ a $300 \mathrm{~mm}$ dado que al ser formas más complejas el material no logra moldearse al formato base estimado.

Por otro lado, al someter a prueba los tejidos de forma circular versus los tejidos cuadrados, los primeros, al no estar tejidos por una fibra continua, tienden a separarse en los puntos donde se superponen las fibras, imposibilitando un movimiento uniforme de ésta para generar las morfologías. En cambio, los tejidos de trama $1 \times 1$ (cuadrados) logran configurar con éxito aquellas probetas conformadas por ángulos entre $20^{\circ}$ y $50^{\circ}$.

De aquí se determina que, para la realización de las pruebas de los grupos restantes, sólo se haga uso del formato de tejido $1 \times 1$ puesto que fue el tejido que logró adaptarse de mejor manera a los moldes.

Para los grupos $E$ y $F$, las probetas con mayor éxito son nuevamente las curvas con ángulos de $50^{\circ}$, siendo esta vez los mejores tejidos aquellos que presentan la trama en contra de la curva, esto quiere decir que será la urdimbre la encargada de generar la curva principal y que la trama es la que debe adaptarse a la transición de la forma.

Finalmente, para el grupo G (el grupo que consta de las morfologías más complejas), se obtiene que el entramado de huira gruesa (> $3 \mathrm{~mm}$ ) no es capaz de moldear las figuras dado que no es lo suficientemente flexible para adaptarse a ellas. En cambio, el entramado de huira fina $(<2 \mathrm{~mm})$ logra conformar con éxito las probetas hasta los $50^{\circ}$, comprobado entonces que, a mayor complejidad de la forma, menor deberá ser la anchura de la fibra.

Grupo B
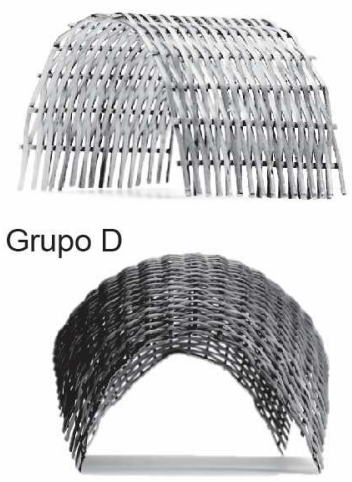

Grupo F

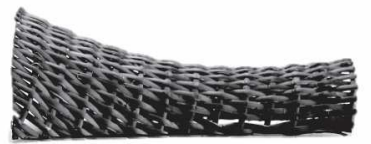

Figura 7: Mejores muestras por grupo. Elaboración propia.

Grupo G

\section{Prototipo Final}

Concluido el análisis de la etapa 1 y 2 se procede a la creación de un prototipo de mayor tamaño que las muestras, utilizando el máximo tamaño de paño posible $(1 \mathrm{~m} \times 1 \mathrm{~m})$ sin superponer fibras, con el propósito de comprobar el aumento de plasticidad que mostró el material durante la ejecución del experimento. Para esto, se combinan los mejores resultados de cada grupo de morfologías, siendo predominante las curvaturas en ángulos de $50^{\circ}$ con un tejido $1 \times 1$ y las formas más complejas (aquellas generadas en el grupo de paraboloide elíptico y en el paraboloide hiperbólico por revolución). Adicionalmente, los encuentros que generan vértices se corrigen por curvas suaves para evitar el quiebre 0 la separación de las fibras al momento de ser sometidas a presión.

La figura 8 muestra el prototipo final una vez desmoldado y sin excedentes de adhesivo, quedando demostrado que es posible la construcción de formas complejas sin la necesidad de estructuras de soporte.

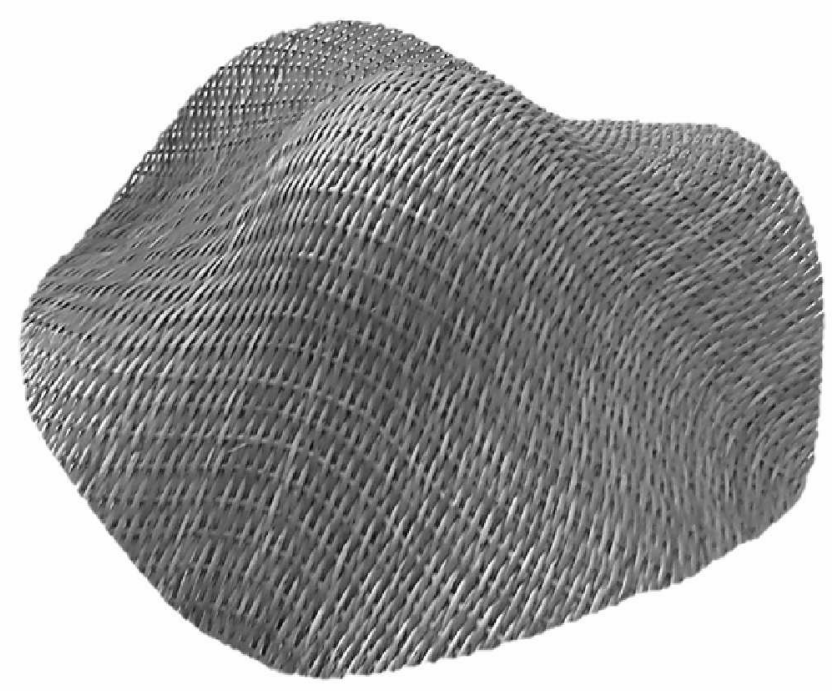

Figura 8: Prototipo final. Elaboración propia
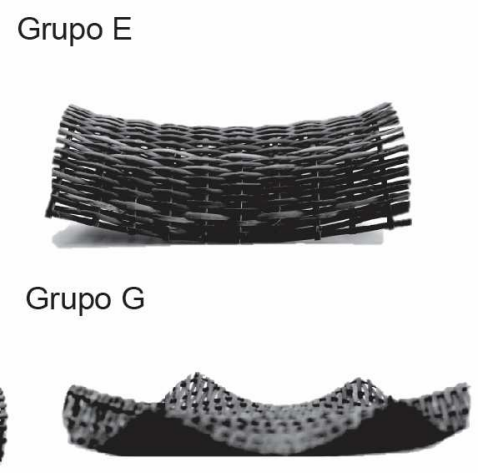

\section{Conclusiones}

El estudio permitió la creación de un método procedimental para la elaboración de un material compuesto de mimbre en forma de huira entramada y de un adhesivo de fijación (acetato de polivinilo). De igual manera, sentó las bases para innovar en los procesos productivos del mimbre a través de la extrapolación de la técnica de machihembrado, lográndose la elaboración de morfologías auto-estructurantes simples y complejas.

Con respecto a la experimentación, ésta logra dilucidar las cualidades principales de la combinación de materiales, permitiendo conocer el comportamiento de éstos durante las distintas etapas morfológicas planteadas. Al mismo tiempo, responde positivamente a la hipótesis expuesta pese a que se 
obtuvieron morfologías con poco éxito, como aquellas con curvaturas pronunciadas $\left(70^{\circ}\right)$, sin embargo, éstas resultan escasas en comparación con aquellas que sí tuvieron éxito, dejando claro que el mimbre combinado con un adhesivo es capaz de aumentar su plasticidad, logrando estructurarlo y fijando su forma.

Si bien las propiedades del mimbre en cuanto a plasticidad y rigidez se ven aumentadas por la combinación con PVA, se entiende que el material no es apto para soportar cargas pesadas. No obstante, la posibilidad de generar morfologías libres de estructuras, complejas en forma y livianas en peso, da la oportunidad de usar el material en nuevos nichos, por tanto, sería interesante ver en el mercado actual planchas o láminas de huira entramada comercializándose como un símil de los tableros existentes, y que al ser sometidas al estudio aquí presentado se puedan generar piezas que, por ejemplo, sirvan para el revestimiento arquitectónico. Sin perjuicio de lo anterior, para asegurar un nuevo nicho se hace necesario someter el material a estudios de esfuerzos mecánicos para posicionarlo frente a esta y futuras aplicaciones.

Finalmente, es importante concluir que este estudio contribuye a una micro-industrialización del trabajo en mimbre (de manera que se posicione como un material entramado maleable, ligero y biodegradable) e incorpora al molde como una oportunidad para realzar el valor de la materia prima nacional a través del establecimiento de procesos industriales.

\section{Agradecimientos}

Cabe destacar que esta investigación se desarrolla en el marco del proyecto de título de la autora, donde parte importante de la ejecución de ésta se realiza gracias al aporte de maquinaria y tutorías de la Universidad de Chile, específicamente de la Facultad de Arquitectura y Urbanismo.

De igual forma, se agradece el apoyo constante de Mauricio Tapia, docente de la carrera de diseño industrial y profesor guía a cargo de dirigir la investigación.

Por último, a Maritza Gallardo, por su apoyo en la revisión metodológica, así como su incondicional apoyo emocional y espiritual.

\section{Referencias}

Abalos, M.; INFOR; INTEC-CHILE; PROCHILE. (1998) Mimbre. Wicker. Chile: De la producción al consumo, Santiago: INFOR.

ASIPLA (2005), Estadísticas 1 Trimestre 2015 Industrial del plástico. Retrieved from: http://www.aliplast.org/admin/archivos/est2015.pdf

Bañó H, M. (2010). El diseño industrial: nuevos aspectos funcionales, estéticos y simbólicos de los productos de consumo. Retrieved from:http://dspace.ceu.es/bitstream/10637/3255/1/Ba\%C3\%B1o Hernandez\%2CManuel10-11.pdf

McCann, E. (2012) Escuela de Publicidad U. del Pacífico; "El nuevo consumidor verde". Revista el Sábado, 23, 16- 17 